\title{
Contabilidad Social y Ambiental para la Sostenibilidad y el Proceso Contable: Reflexiones para su Enseñanza-Aprendizaje ${ }^{1}$
}

\section{Social and Environmental Accounting for the Sustainability and the Accounting Process: Teaching-Learning Reflections}

\author{
Norma B. Geba Adamkova ${ }^{2}$, María Laura Catani Becerra ${ }^{3}$
}

\begin{abstract}
RESUMEN
El cumplimiento de los Objetivos del Desarrollo Sostenible requiere de buena información financiera y no financiera de aspectos sociales y ambientales, además de los económicos y financieros tradicionales. Se rescata que se ha investigado en contabilidad social y ambiental con enfoque socio-ambiental, en la República Argentina la contabilidad social y ambiental forma parte de los Contenidos Curriculares Básicos de la carrera de Contador Público, para la International Federation of Accountants el contador tiene un rol protagónico para la sostenibilidad y que según publicaciones de Accountancy Europe los informes financieros y no financieros deben armonizarse e interconectarse.

En aras de ayudar a la sostenibilidad y con avances en el enfoque socio-ambiental, es el objetivo general contribuir a armonizar los informes contables financieros y no financieros. Es el objetivo específico contribuir con un aprendizaje significativo del enfoque socio-ambiental de la contabilidad social y ambiental y del proceso contable para la sostenibilidad.

De deducciones, inducciones, comparaciones y traspolaciones, el desarrollo contiene resultados de adaptaciones realizadas desde lo económico-financiero hacia el enfoque socio-ambiental y una acepción amplia del proceso contable. A modo de triangulación se incluyen resultados de análisis de viabilidad del proceso contable para lo socio-ambiental. Se reflexiona y se infieren conclusiones.

Se infiere que los marcos doctrinarios y normativos contables considerados sin sesgo económico-financiero permiten brindar información económica, financiera, social y ambiental con enfoque financiero y no financiero para la sostenibilidad. Es posible contribuir con un aprendizaje significativo del enfoque socio-ambiental de la contabi-
\end{abstract}

1 Este ensayo se desarrolla en el marco de los Proyectos I+D: E172 y E173, acreditados ante la Universidad Nacional de La Plata, República Argentina.

2 Universidad Nacional de La Plata, Facultad de Ciencias Económicas. Ciudad de La Plata, Provincia de Buenos Aires, República Argentina, normageba@econo.unlp.edu.ar

3 Universidad Nacional de La Plata, Facultad de Ciencias Económicas. Ciudad de La Plata, Provincia de Buenos Aires, República Argentina, laura.catani@econo.unlp.edu.ar 
lidad social y ambiental a partir del enfoque económico-financiero. Se proponen experiencias de enseñanza-aprendizaje. El proceso contable en su acepción amplia permite reconocer que la elaboración de estados contables y su revisión son subprocesos enmarcados en el conocimiento contable. Es posible emitir informes contables financieros y no financieros, interconectados y complementarios para gestionar la sostenibilidad.

Palabras clave: aprendizaje significativo, enfoque socio-ambiental, información no financiera, objetivos del desarrollo sostenible, proceso contable.

Recepción: 24/08/2020. Aprobación: 20/10/2020

\begin{abstract}
Compliance with the Sustainable Development Goals requires good financial and non-financial information on social and environmental aspects, in addition to traditional economic and financial ones. It is recovered that it has been investigated in social and environmental accounting with a socio-environmental approach, in the Argentine Republic social and environmental accounting is part of the Basic Curricular Contents of the Public Accountant career, for the International Federation of Accountants the accountant has a leading role for sustainability and that according to Publications of Accountancy Europe financial and non-financial reports must be harmonized and interconnected.

In order to help sustainability and with advances in the socio-environmental approach, it is the general objective to contribute to harmonize financial and non-financial accounting reports. It is the specific objective to contribute with a meaningful learning of the socio-environmental approach of social and environmental accounting and the accounting process for sustainability.

From deductions, inductions, comparisons and translations, the development contains results of adaptations made from the economic-financial to the socio-environmental approach and a broad conception of the accounting process. Triangulation includes results of feasibility analysis of the accounting process for the socio-environmental. Conclusions are reflected and inferred.

It is inferred that the accounting doctrinal and regulatory frameworks considered without economic-financial bias enable economic, financial, social and environmental information to be provided with a financial and non-financial approach to sustainability. It is possible to contribute with a meaningful learning of the socio-environmental approach to social and environmental accounting from the economic-financial approach. Teaching-learning experiences are proposed. In its broad sense, the accounting process allows us to recognize that the preparation of accounting statements and their review are sub-processes framed in accounting knowledge. It is possible to issue financial and non-financial, interconnected and supplementary accounting reports to manage sustainability.
\end{abstract}

Keywords: accounting process, meaningful learning, non-financial information, socio-environmental approach, sustainable development goals. 


\section{INTRODUCCIÓN}

Distintas iniciativas reconocen la importancia de la buena información financiera y no financiera de aspectos sociales y ambientales, además de los económicos y financieros tradicionales, para el cumplimiento de los Objetivos del Desarrollo Sostenible (ODS). Las organizaciones económicas, como actores sociales, necesitan de información más holística en aras de gestionarse sosteniblemente. Diversas disciplinas científicas pueden aportar a la sostenibilidad y entre tales se encuentran la ciencia de la administración, la economía y la contabilidad.

Históricamente, los marcos teóricos doctrinarios y normativos contables han contribuido para que las organizaciones económicas obtengan y brinden informes homogéneos sobre su patrimonio e impactos, positivos y negativos, centrados en lo financiero, o enfoque económico-financiero, pero en la actualidad se reconoce que los mismos son insuficientes para gestionar la sostenibilidad organizacional.

Por medio de investigaciones contables sistemáticas, enmarcadas en proyectos de investigación y desarrollo (I+D), a partir del sentido amplio de los conceptos y definiciones contables (sin sesgo económico-financiero), se han realizado adecuaciones y actualizaciones que han permitido desarrollos conceptuales con un enfoque o perspectiva socio-ambiental, no centrada en lo financiero y enmarcada en los objetivos del desarrollo sostenible. Se han distinguido, al menos, dos especialidades contables complementarias: la especialidad económico-financiera y la social y ambiental inclusiva de un enfoque socio-ambiental, además de económico-financiero.

Como una manera de aplicar tales desarrollos, se han seleccionado las memorias de sostenibilidad de la Global Reporting Initiative (GRI). Se ha inferido que los profesionales contables, así como instrumentan en las organizaciones económicas los conocimientos contables con enfoque económico-financiero, podrían instrumentar el enfoque socio-ambiental para la especialidad contable social y ambiental adaptando, sin perder sus especificidades, el proceso contable tradicional, o instaurado en las organizaciones económicas.

En el año 2017 desde el Ministerio de Educación de la República Argentina, por medio de la Resolución 3400-E/2017, se aprueban los contenidos curriculares básicos para acreditar la carrera correspondiente al título de Contador Público. El Anexo I brinda los Contenidos Curriculares Básicos y dentro del Área Temática: Contabilidad e Impuestos se incluye a la contabilidad social y ambiental, entre otros contenidos.

De una comunicación de la International Federation of Accountants (IFAC) en febrero 2020, se interpreta de Stathis Gould (2019) que la visión de IFAC es que la profesión contable es esencial tanto para las organizaciones y los mercados financieros como para las economías fuertes y sostenibles. La "profesión contable tiene un papel crucial en la agenda 2030 para el desarrollo sostenible, como lo representan los Objetivos de Desarrollo Sostenible (ODS) destinados a sostener a las personas, el planeta y la prosperidad."

En diciembre de 2019 la página de Accountancy Europe informa de la publicación Establecimiento de estándares interconectados para informes corporativos, de la cual se interpreta: "Los riesgos 
y oportunidades globales significan que la información financiera por sí sola no puede brindar una imagen completa del desempeño de una empresa", que el "cambio climático, la degradación ambiental, el malestar social y los intangibles generados internamente se abordan mediante informes de información no financiera (NFI)" y que "los cientos de iniciativas de informes de NFI disponibles están generando confusión y la posibilidad de un lavado verde." Se entiende que para "una respuesta eficaz a estos problemas globales y demandas de las partes interesadas, los informes... deben armonizarse e interconectarse con los informes financieros." (Accountancy Europe, 2019). En junio 2020 Accountancy Europe emite: Follow-up paper: Interconnected standard setting for corporate reporting Feedback analysis, del mismo se entiende que: "El objetivo final debería ser una solución de sistema para ofrecer estándares globales de informes NFI conectados a la información financiera." (Accountancy Europe, 2020).

Si bien los marcos contables doctrinarios y normativos son importantes para elaborar informes homogéneos, no es de menor importancia un adecuado proceso contable para su correcta aplicación, para la disminución de riesgos de la operacionalización de los datos (por ejemplo de omisión y duplicación) y permitir que la información brindada por las organizaciones económicas sea metódica, sistemática, mayormente cuantificable y verificable.

De considerar el papel crucial otorgado a la profesión contable en la agenda 2030 para el desarrollo sostenible, el reconocimiento de que gran cantidad de iniciativas de informes no financieros generan o pueden generar confusión y hasta la "posibilidad de un lavado verde", que los informes no financieros deben armonizarse con los informes financieros, que en Argentina la contabilidad social y ambiental se encuentra dentro de los Contenidos Curriculares Básicos del título de Contador Público y que se han realizado adecuaciones y actualizaciones que han permitido desarrollos con un enfoque o perspectiva socio-ambiental, no centrada en lo financiero y enmarcada en los objetivos del desarrollo sostenible, surgen diversos interrogantes, entre ellos: ¿Cómo contribuir a armonizar los informes contables financieros y no financieros para la sostenibilidad? ¿Cómo contribuir a la enseñanza-aprendizaje del enfoque socio-ambiental de la especialidad contable, o segmento, social y ambiental y de adaptaciones del proceso contable para la sostenibilidad?

Si bien se entiende apropiado contar con un marco normativo social y ambiental para la sostenibilidad, transmitir la temática y avances generados para el enfoque socio-ambiental de la contabilidad social y ambiental puede contribuir a armonizar los informes contables financieros y no financieros para la sostenibilidad. De rescatar que el aprendizaje significativo es relevante para las diversas disciplinas y que: "Según Ausubel el aprendizaje significativo requiere: 1. Materiales de aprendizaje significativos"; “2. Una disposición por parte de la persona que aprende a enlazar cada concepto del nuevo material con conceptos que ya tiene"; y "3. Una estructura cognitiva relevante o apropiada en el alumno, es decir, que algunos conceptos de la misma puedan ser relacionados, de manera no arbitraria, con los nuevos conceptos" (González García, 1992, p. 149): se interpreta que una forma de contribuir a un aprendizaje significativo del enfoque socio-ambiental y de adaptaciones del proceso contable para la sostenibilidad es a partir de lo conocido sobre lo económico-financiero tradicional.

Es el objetivo general contribuir a armonizar los informes contables financieros y no financieros para la sostenibilidad. Es el objetivo específico contribuir con un aprendizaje significativo 
del enfoque socio-ambiental de la contabilidad social y ambiental y del proceso contable para la sostenibilidad a partir del enfoque económico-financiero tradicional.

\section{METOLOGÍA}

Se buscan, seleccionan y plasman aspectos relevantes de avances realizados sobre el enfoque socio-ambiental de la denominada especialidad contable social y ambiental. El desarrollo contiene los objetivos del desarrollo sostenible, un marco conceptual básico de la contabilidad como disciplina, adaptaciones realizadas desde el enfoque contable económico-financiero hacia el enfoque socio-ambiental a partir de deducciones, inducciones y comparaciones, sumando traspolaciones desde el proceso contable tradicional hacia el enfoque socio-ambiental. Se incluyen variables relevantes, conceptos y definiciones de activo, pasivo y patrimonio neto sociales y ambientales, entre otras. A modo de triangulación, se complementa con resultados de análisis realizados sobre la viabilidad del proceso contable tradicional para lo socio-ambiental considerando las memorias de sostenibilidad de la Global Reporting Initiative. Se reflexiona y se infieren conclusiones.

\section{DESARROLLO}

Los Objetivos del Desarrollo Sostenible (ODS) propenden a la sostenibilidad económica, social y ambiental, o natural, y reconocen la importancia de su información. Según la página de Naciones unidas, los ODS se componen por 17 objetivos (1. Fin de la Pobreza; 2. Hambre Cero; 3. Salud y Bienestar; 4. Educación de Calidad; 5. Igualdad de Género; 6. Agua Limpia y Saneamiento; 7. Energía Asequible y No Contaminante; 8. Trabajo Decente y Crecimiento Económico; 9. Industria, Innovación e Infraestructura; 10. Reducción de las Desigualdades; 11. Ciudades y Comunidades Sostenibles; 12. Producción y Consumo Responsables; 13. Acción por el Clima; 14. Vida Submarina; 15. Vida de Ecosistemas Terrestres; 16. Paz, Justicia e Instituciones Sólidas; y 17. Alianzas para Lograr los Objetivos) y 169 metas. (Naciones unidas, s.f.).

La Global Reporting Initiatie (GRI), United Nation, Global Compact, y Consejo Empresarial Mundial para el Desarrollo Sostenible (WBCSD) (s.f.), incluyen un pedido a las empresas para que: "evalúen su impacto, establezcan metas ambiciosas y comuniquen de forma transparente sus resultados. Ban Ki-moon”... (p. 4). En el año 2016 se publican los Estándares GRI, proponiéndose su vigencia obligatoria para los informes publicados a partir del 1 de julio de 2018. Los Estándares se desarrollan por el Global Sustainability Standards Board (GSSB) y están integrados por: Estándares Universales Serie 100 y los Estándares temáticos series 200 (temas económicos), 300 (temas ambientales) y 400 (temas sociales). (GSSB, GRI, 2016). Los Estándares temáticos se centran en impactos económicos, ambientales y sociales relevantes vinculados a los ODS y en distintas unidades de medida pertinentes, pero no en el patrimonio económico-financiero-socialambiental.

Amerita considerar que, históricamente los conocimientos contables brindan información metódica, sistemática, mayormente cuantificable y evaluable del patrimonio de las organizaciones (diferenciándolo de los bienes de terceros), sus variaciones, impactos recibidos y generados, y sobre determinados riesgos. Se interpreta que: 
"el fin último" de la contabilidad no es registrar metódicamente algunos hechos de la realidad social (técnica); ni tampoco elaborar o diseñar normas prescriptivas sobre la confección de Estados Contables para resolver cuestiones de hecho (tecnología social); sino valerse de los informes contables elaborados, normalizados o no, para obtener conocimientos metódicos y sistemáticos de la porción de la realidad considerada. (Geba y Fernández Lorenzo, 2001, p. 111).

Puede entenderse a la contabilidad como una "Disciplina científica social cuyo objetivo es brindar información metódica y sistemática, principalmente cuantificada y verificable, sobre el patrimonio de un ente y sus variaciones, destinada a distintos usuarios que toman decisiones en base a la misma." (Lexinómicas, 2012). Para Geba y Bifaretti (2014) la "tecnología contable abarcaría al conjunto de técnicas, conocimientos y procesos que sirven para diseñar y construir objetos (p. e.: informes) para satisfacer necesidades humanas", pudiendo referirse a objetos (hadware contable) y sistemas (SIC); la "actividad tecnológica contable se correspondería con el desarrollo, el diseño, la ejecución y el producto resultante... permitiría disponer de sistemas para comunicarnos, fabricar nuevos objetos, otros"; la motivación en la "contabilidad como ciencia (o disciplina científica) respondería al deseo de conocer y comprender racionalmente, su actividad es la investigación y su producto el conocimiento científico"; “Existiría relación de complementariedad entre la disciplina contable y la tecnología contable" (p. 15).

En las organizaciones económicas la instrumentación y aplicación de los conocimientos y normativas contables requiere de un adecuado proceso contable. Dicho proceso contable debe permitir obtener y brindar informes contables homogéneos que respeten los atributos de la información contable, y, por ende, sean comparables y acumulables. Se han elaborado esquemas de una entendida estructura interna de la disciplina contable y de integrar distintos desarrollos (Fernández Lorenzo y Geba, 2007, Fernández Lorenzo, 2013) es posible elaborar el esquema de la siguiente figura. 


\section{Figura 1}

Una Estructura interna de la disciplina contable

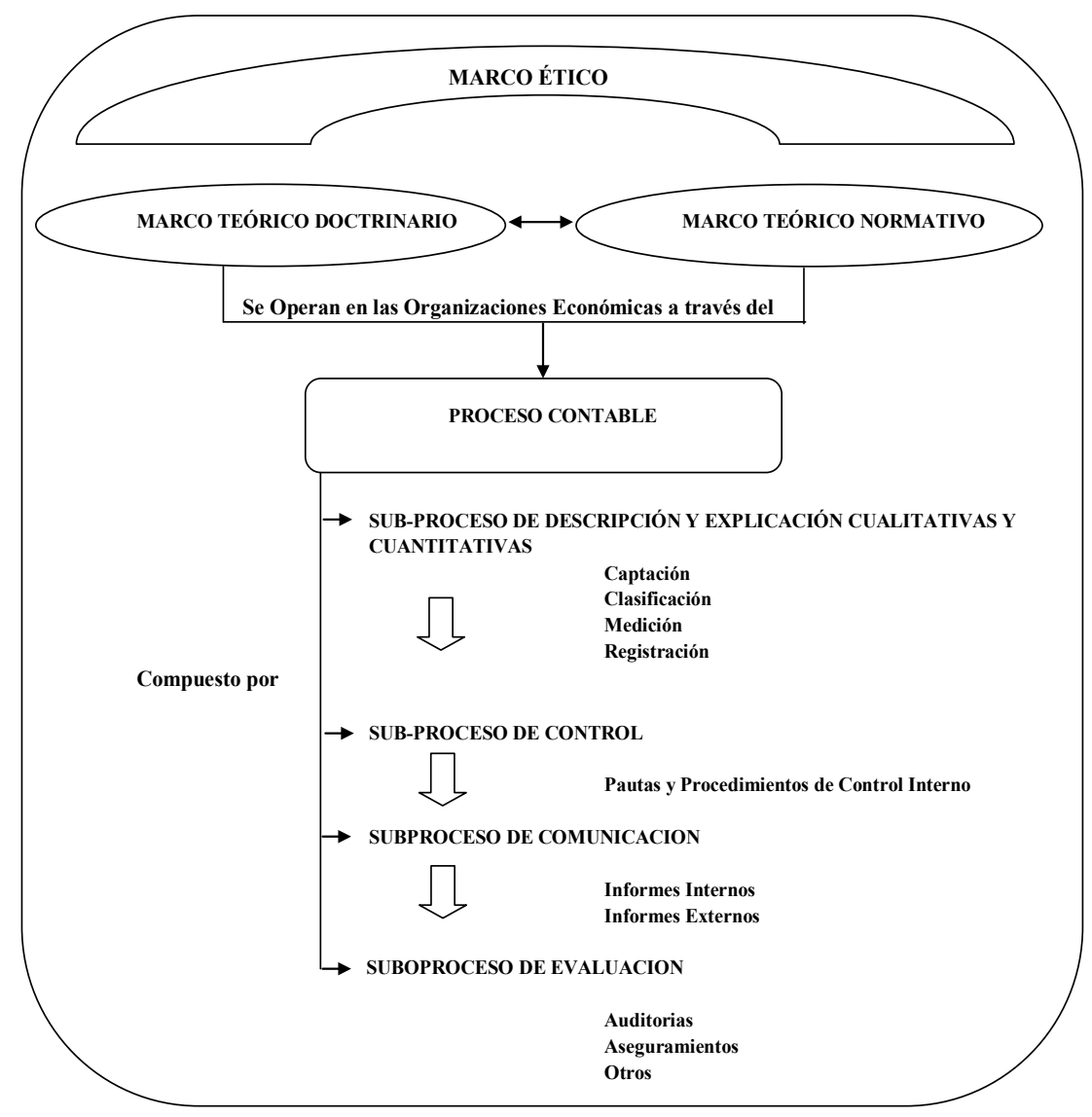

Nota: puede apreciarse una relación entre los marcos contables y el proceso contable en su acepción amplia. Fuente: elaboración propia a partir de Geba (2005) y Fernández Lorenzo (2013).

Para la precedente estructura de la disciplina contable se han considerado el sentido amplio de los términos, el proceso contable un una acepción amplia, y que el marco teórico doctrinario contable nutre, puede nutrir y es nutrido por el marco teórico normativo, el cual se instrumenta, o puede instrumentarse, en las organizaciones económicas por medio de un proceso contable. El proceso contable para el enfoque económico-financiero forma parte del sistema de información de las organizaciones económicas. Diversos autores entienden al proceso contable formado por etapas sin considerar al control y la verificación como partes del mismo. Pero, en una acepción amplia es posible entenderlo constituido por varios sub-procesos relacionados que incluyen al subproceso de control y al de revisión, auditoría, aseguramiento y similares. La acepción amplia del proceso contable contribuye a armonizar su adaptación para lo socio-ambiental, desde la captación del dato hasta la verificación de la información y del proceso.

Se ha interpretado "a la contabilidad como disciplina social con la capacidad de generar modelos contables para diagnosticar y medir el grado de desarrollo humano sustentable en un mo- 
mento y lugar determinados." Para aumentar "la contribución social de la Contabilidad" se ha considerado "un enfoque amplio de los conceptos de riqueza y patrimonio." Los "mismos contienen diferentes dimensiones sociales, tales como: cultura, en sentido subjetivo, salud, trabajo, medioambiente, etc., que pueden ser consideradas dentro de un 'marco contable' de manera similar a la económica- financiera tradicional." (Geba y Sebastián, 2002, p. 3). Se han analizado y trabajado las variables básicas del modelo económico-financiero para lo socio-ambiental, no centrado en lo financiero. Como resultado de ello, se han elaborado distintas figuras, entre las cuales se encuentra la siguiente:

\section{Figura $N^{0} 2$}

Del enfoque, o modelo, contable económico-financiero al enfoque socio-ambiental

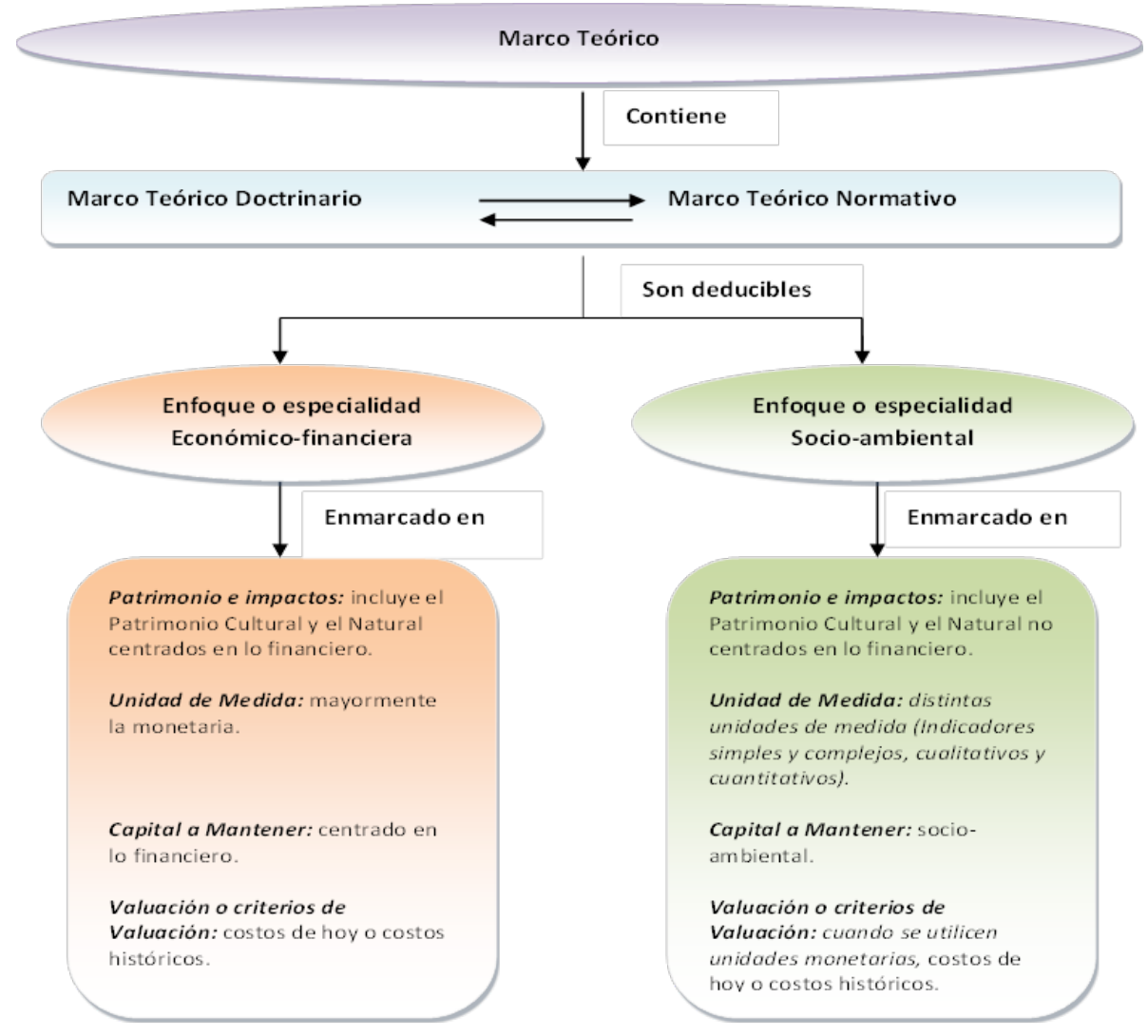

Nota: es posible observar las variables relevantes del modelo económico-financiero para lo socio-ambiental. Fuente: Geba, Catani y Sebastián (2018).

Si bien ciertos aspectos sociales y ambientales pueden ser estudiados con un enfoque económico-financiero, en la Figura $\mathrm{N}^{\mathrm{o}} 2$ puede observarse una forma de adaptar las variables del modelo contable económico-financiero para el enfoque, o perspectiva, socio-ambiental de aspectos sociales y ambientales no centrados en lo financiero. La mencionada adaptación, también ha permitido confirmar como: "Objetivo de la contabilidad socio-ambiental: obtener y brindar conocimiento racional de la dimensión socio-ambiental considerada, es decir del patrimonio socio-ambiental, 
los impactos socio-ambientales y sus relaciones, no meramente financieros, en el ente, sujeto del proceso, y en su entorno"... (Geba, Fernández Lorenzo y Bifaretti, 2007, p. 9).

Conocer la composición cualitativa y cuantitativa del patrimonio de las organizaciones económicas con enfoque económico-financiero y social-ambiental, a un momento y lugar determinados, permitiría contribuir a determinar la materialidad de los impactos y los recursos propiedad del ente para afrontar sus obligaciones hacia terceros, así como la proporción de dichos recursos que son aportados por los propietarios. Se interpreta que la adaptación, o adecuación, realizada a los términos contables tradicionales propiciaría procesar metódicamente datos concretos, obtener y emitir informes homogéneos con enfoque socio-ambiental con atributos de los estados contables financieros. Desde el proceso contable con enfoque económico-financiero, en su acepción amplia, se desarrollan aspectos básicos del proceso contable para el enfoque socio-ambiental en el marco de la entendida especialidad social y ambiental, propiciando su utilización para ambos enfoques.

\section{Figura 3}

El proceso contable desde lo económico-financiero hacia lo socio-ambiental

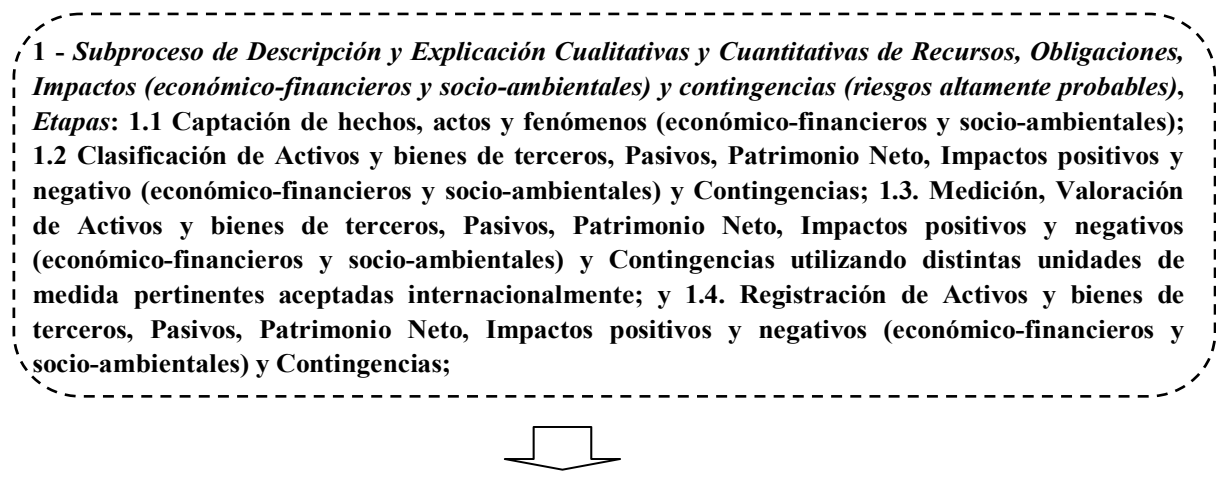

2 - Subproceso de control: pautas y procedimientos de control que pueden ser anteriores, concomitantes y/o posteriores a cada etapa o circunstancia económico-financiera y socio-ambiental a controlar;

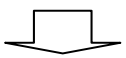

3 - Subproceso de comunicación referido a Recursos (Activos y bienes de terceros), Obligaciones (Pasivo y Patrimonio Neto), Impactos positivos y negativos (económico-financieros y socio-ambientales) y Contingencias: Exponer o comunicar información contable (económico-financiera y social y ambiental) a través de informes internos y externos que cumplan los atributos de la información contable.

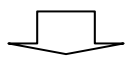

4 - Subproceso de Evaluación referido a la información económica-financiera y socio-ambiental y a los procesos: pueden incluirse a las Auditorias, Aseguramientos y otros pertinentes.

Nota: se plasman componentes de los subprocesos contables para el enfoque económico-financiero y el socio-ambiental. Fuente: elaboración propia a partir de Geba, Fernández Lorenzo y Sebastián (2007) y Fernández Lorenzo (2013). 
Si bien en la práctica quien audita externamente, tanto la información como su proceso, no deben pertenecer al ente titular de la información o su proceso, para el proceso contable que se plasma en la Figura $\mathrm{N}^{\mathrm{o}} 3$ se han considerado a los sub-procesos en su conjunto como una manera de analizar y observar que se enmarcan en normativas contables y que el proceso contable para lo socio-ambiental puede ser similar al utilizado para lo económico-financiero tradicional.

En cuanto a los reportes de sustentabilidad empresaria y su auditoría, en un estudio que considera empresas chilenas incluidas dentro del Índice de Precio Selectivo de Acciones (IPSA) de la Bolsa de Comercio de Santiago se "analiza si la presentación de reportes de sustentabilidad y su auditoría tienen efecto... en la liquidez de mercado", se concluye "que aquellas empresas que emiten reportes de sustentabilidad tienen un efecto positivo en la liquidez de mercado." (Zúñiga Pérez, Pincheira Lucas, Aguilar Cárcamo y Silva-Briceño, 2020, p. 63).

De una analogía entre los atributos de la información de los estados contables financieros y los principios de calidad de las memorias de sostenibilidad según los Estándares de la Global Reporting Initiative surgen ciertas similitudes, excepto por el atributo de sistematicidad que es exigible para los estados contables.

De tener en cuenta que las memorias de sostenibilidad de la Global Reporting Initiative conforman el Balance Social para Argentina, que los Estándares 2016 de la Global Reporting Initiative no son una nueva versión de G4 y se encuentran vinculados a los objetivos del desarrollo sostenible, se rescatan los siguientes análisis de viabilidad del proceso contable y sus sub-procesos componentes para el enfoque socio-ambiental.

De los análisis realizados se ha observado para lo socio-ambiental que en 1- Subproceso de Descripción y Explicación Cualitativas y Cuantitativas de Recursos, Obligaciones, Impactos (económicofinancieros y socio-ambientales) y contingencias, Etapa 1. Captación: según Geba, Bifaretti y Sebastián (2014) "para más del 52\% de los indicadores ambientales de G4 el SIC instaurado en las organizaciones económicas puede aportar a través de un proceso contable homogéneo... a la elaboración del Balance Social, incorporando las variables ambientales incluidas en dicha versión." Se ha interpretado que dicho "proceso contable contribuirá también con la verificación de la transformación del dato en información, de manera de evitar riesgos de duplicaciones o, incluso, inconsistencias u omisiones." (p. 15).

En Catani (2016) se realiza un análisis crítico sobre la Categoría Dimensión Social de la versión G4 y el 100\% de sus aspectos, se seleccionan "el 72,92\% de sus indicadores para los cuales se encontraría, o podrían incluirse datos o adaptarse" la documentación respaldatoria en el Sistema de Información Contable (SIC) instaurado en las organizaciones económicas. (p. 2). Surge que "23 pueden ser elaborados con datos que se encuentran en la documentación que se procesa actualmente en el SIC"; " 5 requieren de datos base que se encuentran en el SIC pero necesitan ser ampliados por grupos de interés internos; y 7 necesitan de datos base que se encuentran en la documentación procesada en el SIC pero requieren ser ampliados por grupos de interés externos." (p. 3). Se reconoce que la "adaptación/modificación del SIC de la organización tiene importancia por el indicador en sí mismo, pero también presenta un valor agregado, ya que puede brindar más y mejores conocimientos y ayudar al ejercicio, medición y evaluación de la gestión organizacional." (p. 3). 
Para la Etapa 2. Clasificación: desde el enfoque económico-financiero se ha analizado y distinguido un entendido activo para lo social y ambiental, al igual que el pasivo para social y ambiental y el capital social y ambiental, enmarcados en la sostenibilidad. Se ha interpretado que impactos o resultados positivos sociales y ambientales provocan aumentos en el activo social y ambiental, natural, o disminuciones en el pasivo social y ambiental respectivamente, mientras que los resultados negativos disminuyen el activo o aumentan el pasivo. Se ha inferido que el enfoque contable socio-ambiental contribuiría a reconocer el capital social y ambiental del ente (el cual podría ser relacionado y comparado con los límites mínimos a mantener de recursos ambientales o sociales del contexto) y partidas de ingreso y de gastos sociales y ambientales de un periodo. Para la elaboración sistemática de un plan de cuentas básico, se han interpretado, entre otros:

Activo natural (ambiental) no dependería de su tangibilidad, de la forma de adquisición, de la posibilidad de venderlo por separado, de la erogación previa de un costo. Es decir, un elemento activo natural haría referencia a bienes y servicios ambientales, o stocks de la naturaleza que producen una renta natural, o flujo de bienes y servicios que son útiles (p.ej.: una población de árboles que autogenera nuevos árboles y otros servicios ecosistémicos - de soporte y estructuras, de regulación atmosférica, climática, hídrica, formación del suelo, regulación de nutrientes, control biológico y culturales como la mejora emocional y percepción), incorporado al patrimonio (por compra, donación recibida, aporte de los propietarios, autogeneración, otras), con o sin erogación previa. Asimismo, la posibilidad de flujos positivos de un activo natural debería estar asegurada con certeza (ciertos) o esperada con un alto grado de probabilidad (probables), pudiendo ser directa o indirecta.

... En cuanto a la exposición, los activos naturales, en un principio podrían incluirse como bienes de cambio o bienes de uso, y como activo corriente o no corriente según corresponda. Adicionalmente, debería emitirse en la información complementaria la composición de los rubros, así como el grado de incertidumbre asociado al proceso de medición de flujos futuros netos esperados en función de la naturaleza, con y sin intervención humana. (Geba, Bifaretti y Sebastián, 2013, p. 22).

Los activos naturales comprenderían, cuando correspondan, aspectos del agua, la tierra, el aire, biodiversidad, otros. (Geba y Gastañaga, 2014, p. 7).

Activo Social: no debería depender de su tangibilidad, ni de la forma de adquisición, ni de la posibilidad de venderlo por separado, ni de la erogación previa de un costo. Es decir, un elemento activo social haría referencia a bienes y servicios sociales en concordancia con la responsabilidad social de la organización, la riqueza social o el grado de desarrollo social de una comunidad. (Bifaretti y Catani, 2015, p. 18).

Pasivo ambiental-natural: estaría conformado cuando por un hecho ocurrido el ente está obligado a entregar activos o prestar servicios, o es altamente probable que ello ocurra, y su cancelación es ineludible. Abarcaría las obligaciones legales como las asumidas voluntariamente, cuando del comportamiento del ente puede deducirse que aceptará ciertas responsabilidades frente a terceros, creando expectativas de que cancelará esa obligación mediante la entrega de activos o la prestación de servicios... de rescatar que en el marco del desarrollo sustentable se 'apunta ' a 
minimizar el pasivo ambiental o, por lo menos a evitar que se incremente, el pasivo ambiental podría estar generado por [obligaciones normativas o compromisos asumidos para evitar daños ambientales] (contaminación del agua, del suelo, del aire, deterioro de los recursos y de los ecosistemas, otros) producidos por un hecho anterior y que superen [o no] los límites para mantener un determinado equilibrio ecosistémico, el que podría estar determinado por procedimientos de evaluación de impacto ambiental previos y referidos a los daños ambientales generados, o a generar, que representen un daño o un riesgo actual o potencial significativo. Cuantitativamente, se podrían utilizar distintas unidades de medida, indicadores simples y complejos, pertinentes... los pasivos naturales podrían incluir la dimensión natural comprometida (agua, tierra, aire, biodiversidad, otras), y como pasivo corriente o no corriente según corresponda. Adicionalmente, debería emitirse en la información complementaria la composición de los rubros, así como el grado de incertidumbre asociado al proceso de medición. (Geba, Bifaretti y Sebastián, 2013, p. 22).

Pasivo social-sociedad: podría estar conformado cuando por un hecho anterior el ente está obligado (legalmente o voluntariamente al aceptar determinadas responsabilidades hacia terceros) a transferir activos o prestar servicios, o es altamente probable que así sea y su cancelación no puede eludirse. (Bifaretti y Catani, 2015, p. 18).

En Argentina se diferencia Patrimonio de Patrimonio Neto, de ello puede entenderse que el:

Patrimonio neto natural: resultaría del aporte de los propietarios o asociados y de la acumulación de resultados netos naturales. En este caso puede resultar: Patrimonio neto natural $=$ Activo natural - Pasivo natural... el patrimonio neto natural estaría conformado por un capital natural (que no debería ser inferior al "capital natural mínimo a mantener" para preservar el equilibrio ecosistémico) y los resultados netos naturales de los distintos ejercicios económicos. (Geba, Bifaretti y Sebastián, 2013, p. 23). Resultado natural del período: habría una denominada ganancia o superávit cuando aumenta el patrimonio natural y una pérdida o déficit en el caso contrario [no originados en las relaciones del ente con los propietarios]. (p. 23). Ingresos naturales: aumentos del patrimonio neto natural originados por la regeneración natural o por servicios ecosistémicos, con o sin la intervención humana [como el crecimiento natural o inducido de determinados activos vegetales o animales, la generación de energía, otros]... Gastos naturales: disminuciones del patrimonio neto natural relacionadas de manera menos directa que los costos [consumidos]... (Geba, Bifaretti y Sebastián, 2013, p. 23).

Mientras que el "Patrimonio neto social: resultaría del compromiso y/o del aporte de los propietarios o asociados y de la acumulación de resultados netos sociales"; y el "Resultado social del período: podría generarse por aumento o disminución del patrimonio social no originado en las relaciones del ente con sus propietarios." (Bifaretti y Catani, 2015, p. 18).

Para la Etapa 3 Medición, Valoración: se ha interpretado que pueden utilizarse distintas unidades de medida pertinentes, incluida la monetaria. Como es necesario que las unidades de medidas empleadas sean inalterables, universales y de fácil reproducción, se han recomendado las dis- 
puestas por los organismos internacionales referentes. Para el análisis de viabilidad del proceso contable se han utilizado las unidades de medida consideradas para las memorias GRI.

En la Etapa 4. Registración de Recursos (Activos y bienes de terceros), Obligaciones (Pasivo y Patrimonio Neto), Impactos positivos y negativos (económico-financieros y socio-ambientales) y contingencias (sociales y ambientales), las organizaciones podrían, de manera similar a los registros utilizados para lo económico-financiero, usar registros contables para lo social y ambiental aplicando el método de la partida doble, siguiendo la rutina implementada al efecto y apoyadas por la tecnología. Se ha entendido conveniente diferenciar los bienes que forman parte del Activo social y ambiental de los bienes de terceros para contribuir a evitar lo interpretado como "lavado de activos".

Ha sido posible analizar el subproceso de control, el de comunicación y el de evaluación considerando aspectos doctrinarios, normativas contables y destacados referentes. Para el subproceso de comunicación, de los análisis realizados sobre informes o memorias de sostenibilidad, es posible expresar que dichos informes contienen información económica-financiera-social-ambiental y en distintas unidades de medidas sobre los impactos incluidos y relacionados a los objetivos del desarrollo sostenible (ODS), la cual es meritoria y puede ser útil para determinar resultados sociales y ambientales.

Según Geba y Giusso (2017) es “posible afirmar que el proceso contable es útil para cubrir información enmarcada por los Estándares GRI y contribuir a conocer y optimizar la implementación de los ODS en las organizaciones económicas." (p. 23).

\section{CONSIDERACIONES FINALES}

Como resultado del desarrollo plasmado y de las reflexiones realizadas es posible inferir que los marcos doctrinarios y normativos contables considerados en su expresión amplia (sin sesgo económico-financiero) permiten abrir un amplio horizonte y brindar información económica, financiera, social y ambiental (con enfoque financiero y no financiero) para la sostenibilidad.

Como en la especialidad contable social y ambiental pueden utilizarse el enfoque económicofinanciero y el socio-ambiental, es posible contribuir a la enseñanza aprendizaje de la contabilidad social y ambiental con enfoque socio-ambiental a partir de lo económico-financiero tradicionalmente conocido, permitiendo una apropiación sustantiva de los conocimientos, no arbitraria ni mecánica. Para lograr dicha contribución se proponen experiencias de enseñanza-aprendizaje en el aula. Para el aprendizaje significativo pueden observarse similitudes entre el significado sustancial de los términos para lo económico-financiero y para lo socio-ambiental, ya que ambos enfoques respetan la especificidad contable. Con ello, su posibilidad de sistematización con la lógica contable.

Pueden mencionarse entre las principales diferencias que desde el enfoque socio-ambiental es posible distinguir como: Activo social la educación, la salud, los puestos de trabajo, la no discriminación, entre otros; mientras que desde el enfoque económico-financiero suelen interpretarse como un resultado negativo (por lo general gasto). También, desde el enfoque socio-ambiental 
pueden considerarse como Activo ambiental: los derechos de emisión otorgados y controlados, los recursos naturales protegidos, los reservorios, entre otros.

Para el proceso contable de datos que permite la emisión de informes que cumplen los atributos de los estados contables, considerar el proceso contable en su acepción amplia permite reconocer que tanto la elaboración de estados contables como su revisión son procesos, o subprocesos, enmarcados en el conocimiento doctrinario y normativo contable. Es posible observar que la rutina del proceso contable de datos con enfoque económico-financiero puede ser utilizada para el enfoque socio-ambiental, considerando el cambio de enfoque o perspectiva. Es necesario adaptar para lo socio-ambiental los planes de cuenta, los manuales de cuenta y la documentación respaldatoria que se encuentran en el sistema de información del ente.

El procesamiento contable (metódico y sistemático) de datos de la realidad concreta de las organizaciones, enmarcado en los enfoques económico-financiero y socio-ambiental de las especialidades económico-financiera y social y ambiental, permitiría obtener y emitir información financiera y no financiera interconectada y complementaria de aspectos económicos, financieros, sociales y ambientales que cumplan los atributos de la información contable y emanen de un mismo proceso para gestionar la sostenibilidad.

\section{REFERENCIAS BIBLIOGRÁFICAS}

Accountancy Europe (21 de diciembre de 2019). Interconnected standard setting for corporate Reporting. Establecimiento de estándares interconectados para informes corporativos. https: / / www. accountancyeurope.eu / publications / interconnected-standard-setting-for-corporate-reporting/

Accountancy Europe (15 de junio de 2020). Follow-up paper: Interconnected standard setting for corporate reporting Feedback analysis. https: / www.accountancyeurope.eu/publications/follow-up-paper-interconnected-standard-setting-for-corporate-reporting/

Bifaretti, M. y Catani, M. (4-6 de noviembre de 2015). Contabilidad socio-ambiental: conceptos contables relevantes para la dimensión social y los marcos conceptuales normativos [ponencia]. XXXVI Jornadas Universitarias de Contabilidad y V Jornadas Universitarias Internacionales de Contabilidad. Universidad de Buenos Aires, Facultad de Ciencias Económicas, Buenos Aires, República Argentina.

Catani, M., con la colaboración de Giusio, M. y Roellig, I. (11-12 de agosto de 2016). Contabilidad Socio-ambiental: La Documentación Respaldatoria y la Captación de Impactos Sociales para Elaborar Memorias de Sostenibilidad GRI G4 a través del Proceso Contable [ponencia]. $22^{\circ}$ Encuentro Nacional de Investigadores Universitarios del Área Contable. Universidad Nacional de Catamarca, Facultad de Ciencias Económicas y de Administración, Instituto de Investigaciones Contables, San Fernando del Valle de Catamarca, República Argentina.

Fernández Lorenzo, L. y Geba, N. (21-24 de octubre de 2007). Información Contable y Responsabilidad Social Empresarial Activa [trabajo nacional]. XXVII Conferencia Interamericana de Contabilidad. Colegio de Auditores de Bolivia, Asociación Interamericana de Contabilidad (AIC), Santa Cruz de La Sierra, Bolivia.

Fernández Lorenzo, L, con la colaboración de Chianelli, G y Guarracino, A. (2013). El Proceso Contable. En Fernández Lorenzo, L. E., Introducción a la teoría y práctica contables (pp. 77- 
101). La Plata, Argentina: Ediciones Haber Ciencias Económicas.

Geba, N. y Fernández Lorenzo, L. (3-5 de octubre de 2001). Reflexiones sobre el Status Epistemológico de la Contabilidad [ponencia]. VII Jornadas de Epistemología de las Ciencias Económicas. Universidad de Buenos Aires, Facultad de Ciencias Económicas, Buenos Aires, República Argentina.

Geba, N. y Sebastián, M. (30 de agosto de 2002). La necesidad de acrecer los Modelos Contables. Un Análisis de las dimensiones sociales informadas por Empresas lucrativas en sus Estados Contables Tradicionales [ponencia]. $8^{\circ}$ Encuentro Universitario de Investigadores del Área Contable. Universidad Católica de Santa Fe, Facultad de ciencias Económicas, Santa Fe, República Argentina.

Geba, N. (8 de julio de 2005). El Proceso Contable en la Contabilidad como Disciplina [ponencia]. 11mo. Encuentro Nacional de Investigadores Universitarios del Área Contable. Universidad Nacional de Misiones, Facultad de Ciencias Económicas, Instituto de Investigaciones y Estudios Contables, Posadas, República Argentina.

Geba, N., Fernández Lorenzo, L. y Bifaretti, M. (7-9 de noviembre de 2007). Marco Conceptual para la Especialidad Contable Socio-ambiental [ponencia]. XXVIII Jornadas Universitarias de Contabilidad. Universidad Nacional de Mar del Plata, Facultad de Ciencias Económicas y Sociales, Mar del Plata, República Argentina.

Geba, N., Fernández Lorenzo, L. y Sebastián, M. (13-14 de diciembre de 2007). El Proceso Contable en la Especialidad Socio-Ambiental [ponencia]. 13 ${ }^{\text {er }}$ Encuentro Nacional de Investigadores Universitarios del Área Contable y $3^{\text {er }}$ Simposio Regional de Investigación Contable. Universidad Nacional de La Plata, Facultad de Ciencias Económicas, Instituto de Investigaciones y Estudios Contables, La Plata, República Argentina.

Geba, N. Bifaretti, M. y Sebastián, M. (1-3 diciembre de 2013). Reflexiones para elaborar un Marco Conceptual de las Normas Contables Ambientales [trabajo nacional]. XXX Conferencia Interamericana de Contabilidad. Colegio de Contadores, Economistas y Administradores del Uruguay, Asociación Interamericana de Contabilidad (AIC), Punta del Este. República Oriental del Uruguay.

Geba, N. y Bifaretti, M. (11-12 de diciembre de 2014). Diferencias y similitudes entre disciplina contable y tecnología contable [ponencia]. $20^{\circ}$ Encuentro Nacional de Investigadores Universitarios del área Contable y $10^{\circ}$ Simposio Regional de Investigación Contable. Universidad Nacional de La Plata, Facultad de Ciencias Económicas, La Plata, República Argentina.

Geba, N., Bifaretti, M. y Sebastián, M. (24-26 de septiembre de 2014). El Sistema de Información Contable y los Indicadores Ambientales de las Memorias de Sostenibilidad Versión G4 [ponencia]. XXXV Jornadas Universitarias de Contabilidad. Universidad Católica de Cuyo, sede San Juan, Facultad de Ciencias Económicas y Empresariales, San Juan, República Argentina.

Geba, N. y Gastañaga, D. (24-26 de septiembre de 2014). Un estudio exploratorio sobre el patrimonio socio-ambiental y los indicadores ambientales de una memoria de sostenibilidad [ponencia]. XXXV Jornadas Universitarias de Contabilidad. Universidad Católica de Cuyo, sede San Juan, Facultad de Ciencias Económicas y Empresariales, San Juan, República Argentina.

Geba, N. y Giusso, G. (15-17 de noviembre de 2017). Contabilidad Social y Ambiental: Los Estándares GRI 2016 y los Objetivos del Desarrollo Sustentable [ponencia]. XXXVIII Jornadas Universitarias de Contabilidad. Universidad Nacional del Sur, Departamento de Ciencias de la Administración, Bahía Blanca, República Argentina. 
Geba, N., Catani, M. y Sebastián, M. (10-12 de octubre de 2018). Especialidades Contables Económico-Financiera y Social y Ambiental y los Informes de Gestión [ponencia]. VI Jornadas Universitarias Internacionales de Contabilidad y XXXIX Jornadas Universitarias de Contabilidad. Universidad Nacional de Misiones, Facultad de Ciencias Económicas, Puerto Iguazú, República Argentina.

Global Reporting Initiative (GRI), United Nation, Global Compact y Consejo Empresarial Mundial para el Desarrollo Sostenible (WBCSD). (s.f.). SDG Compass La guía para la acción empresarial en los ODS [Archivo PDF]. https:/ / sdgcompass.org/wp-content/uploads/2016 / 06 / SDG_Compass_Spanish-one-pager-view.pdf

González García, F. M. (1992). Los Mapas Conceptuales de J.D. Novak como Instrumentos para la Investigación en Didáctica de las Ciencias Experimentales. Enseñanza de las ciencias: revista de investigación y experiencias didácticas, 10(2), 148-158. www.raco.cat/index.php/Ensenanza/ article/ download/39815/93182

Naciones Unidas (s.f.). Objetivos de Desarrollo Sostenible. https:/ / www.un.org/sustainabledevelopment/es / objetivos-de-desarrollo-sostenible/

Stathis Gould, A. (6 de noviembre de 2019). 5 acciones clave para las organizaciones profesionales de contabilidad (PAO) para promover los ODS. https:/ / www.ifac.org/knowledge-gateway/ contributing-global-economy/discussion/5-key-actions-professional-accountancy

Stichting Global Reporting Initiative (GRI), Global Sustainability Standards Board, Global Reporting Initiative (GSSB, GRI). (2016). GRI Standards Download Center - Traducción al Español (Spanish Translations) [Archivos PDF]. https:/ / www.globalreporting.org/standards / gri-standards-translations / gri-standards-spanish-translations-download-center /

Universidad Nacional de La Plata, Facultad de Ciencias Económicas (octubre-noviembre 2012). Lexinómicas Glosario interdisciplinario. En AU24. http: / / www.au24.econo.unlp.edu.ar/ mod / glossary / vieuw.php?id=1148\&mode=letter\&hook=C\&sortkey=\&sortorder=

Zúñiga Pérez, F. R., Pincheira Lucas, R., Aguilar Cárcamo, J. y Silva Briceño, J. (2020). Informes de sustentabilidad y su auditoría: efecto en la liquidez de mercado chileno. Estudios Gerenciales 36(154), 56-65. https: / / doi.org/10.18046/j.estger.2020.154.3558

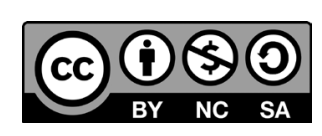

Esta obra está bajo una licencia de

Creative Commons Atribución-NoComercial-CompartirIgual 4.0 Internacional 